\title{
Optical properties of titanium oxycarbide thin films
}

\author{
L. Marques ${ }^{\mathrm{a}}$, H. M. Pinto ${ }^{\mathrm{a}}$, A. C. Fernandes ${ }^{\mathrm{a}}$, O. Banakh ${ }^{\mathrm{b}}$, F. Vaz ${ }^{\mathrm{a}}$, M. M. D. Ramos ${ }^{\mathrm{a}}$ \\ ${ }^{a}$ Universidade do Minho, Departamento de Física, Campus de Gualtar, 4710-057, Braga, Portugal \\ ${ }^{b}$ Haute Ecole ARC Ingénierie, Eplatures-Grise 17, CH-2300 La Chaux-de-Fonds, Switzerland
}

\begin{abstract}
The optical properties of $\mathrm{TiC}_{x} \mathrm{O}_{y}$ thin films, deposited by reactive dc magnetron sputtering at different oxygen flow, were investigated by spectroscopic ellipsometry in the energy range of 0.75-4.5 eV. The dielectric functions measured in the energy range of intraband transitions were analyzed using the classical Drude theory. These results show that free plasma energy and the damping constant of the films depend strongly on film stoichiometry and on their oxygen content. The interband contribution to the optical conductivity of these films is in good agreement with the optical conductivity obtained from first principles calculations based on density functional theory. Both the experimental and the calculated results show that it is possible to significantly modify the optical properties of titanium oxycarbide by adjusting the oxygen content.
\end{abstract}




\section{Introduction}

Transition metal carbides, $\mathrm{MeC}_{\mathrm{x}}$, and oxides, $\mathrm{MeO}_{\mathrm{y}}$, (where Me stands for a transition metal), also known as refractory metal compounds, have been extensively studied during the last decades due to their wide application in industry [1-3]. Separately, they show metallic properties like electrical conductivity and typical properties of covalent/ionic bonded materials such as extreme hardness and high melting point. From a fundamental point of view, their high hardness and melting point are explained in terms of the strong overlap between the anionic $\mathrm{O}(\mathrm{C}) 2 p$ orbital with $d$ electrons of the transition metal. For example, the good conductivity of $\mathrm{TiO}$ is a result of the high occupation of the Ti 3d bands close to the Fermi level. Taking into account the physical properties of $\mathrm{TiC}$ and $\mathrm{TiO}$ compounds, the possibility of growing single phase $\mathrm{TiC}_{1-\mathrm{x}} \mathrm{O}_{\mathrm{x}}$ or a nanocomposite of $\mathrm{TiC}_{\mathrm{x}}-\mathrm{TiO}_{\mathrm{y}}$ mixed phases appears to be an important issue in order to find new multifunctional coatings based on titanium oxycarbides. Furthermore, little is known about the TiCxOy structures, although these films are known to be homogeneous and to have vacancies on both metal and non-metal sublattices over a wide range of compositions. These structural defects, although may seem undesirable in a first approximation, enable in some cases to obtain a unique combination of properties that may attracted the attention of several different fields of materials $R \& D$. In spite of the remarkable properties shown by this ternary compound, only recently it received the adequate attention in some experimental and theoretical studies [4-5].

In this work we report experimental data on the optical properties of $\mathrm{TiC}_{x} O_{y}$ films, determined by spectroscopic ellipsometry, and these results were analysed using both classical and quantum mechanical theoretical methods. The free electron properties of the compounds were evaluated using the classical Drude model by analyzing the low energy region of the correspondent dielectric functions. The optical properties of these compounds are then correlated to film stoichiometry and compared with the optical properties of 
stoichiometric titanium oxycarbides obtained by first principles calculations, using density functional theory (DFT) [6].

\section{Experimental procedure}

The $\mathrm{TiC}_{\mathrm{x}} \mathrm{O}_{\mathrm{y}}$ films were deposited onto single crystal silicon (100) substrates by reactive dc magnetron sputtering, in a laboratory-size deposition system. It consists of two vertically opposed rectangular magnetrons (unbalanced of type 2), in a closed field configuration. The pumping speed of the deposition system was adjusted to 356 L.s ${ }^{-1}$. Films were prepared with the substrate holder positioned at $70 \mathrm{~mm}$ in all runs and a rotation mode was used (to enhance the homogeneity of the layers), with a constant speed of seven rotations per minute, using a dc density of $100 \mathrm{~A} . \mathrm{m}^{-2}$ on the Ti target $\left(200 \times 100 \mathrm{~mm}^{2}-99.6\right.$ at. \% purity), containing 12 cylindrical carbon pellets (10 mm diameter) placed in the preferential eroded zone. A gas atmosphere composed of argon (working gas) and oxygen (the reactive gas) was used for the depositions. The Ar flow was kept constant at $60 \mathrm{sccm}$. The oxygen flow was adjusted in the range of 0-4 sccm, corresponding to a partial pressure variation from 0 to $3.5 \times 10^{-2} \mathrm{~Pa}$, in order to deposit films with different oxygen contents. The residual pressure inside the

deposition system was $10^{-4} \mathrm{~Pa}$, whereas during the deposition the total pressure was approximately constant at about $0.5 \mathrm{~Pa}$. The coatings were grown with the substrates grounded, and at a temperature close to $200{ }^{\circ} \mathrm{C}$ (an external heating resistance positioned at 80 $\mathrm{mm}$ from the substrate holder was used to heat the samples).

The chemical composition of the coatings was investigated with a Cameca SX-50 Electron Probe Micro Analysis (EPMA), operating at $15 \mathrm{keV}$ as acceleration voltage. The elemental quantification was performed by comparing the peak intensity in the sample and in standards for each element, and applying a ZAF correction to the results. The composition analysis show that the prepared samples lye in the vicinity of sub-stoichiometric $\mathrm{TiC}_{\mathrm{x}}$-type 
compounds, moving to the "surrounding area" of TiO-type films. The film thicknesses varied roughly between 2 and $3 \mu \mathrm{m}$.

The structure and phase distribution of the coatings were accessed by X-ray diffraction (XRD), using a conventional Philips PW 1710 diffractometer, operating with $\mathrm{Cu} \mathrm{K}$ radiation, in a Bragg-Brentano configuration. The XRD patterns were deconvoluted and fitted with a Voigt function to determine the structural characteristics of the films, such as the peak

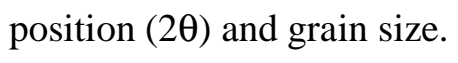

Optical parameters, namely refractive index (n) and extinction coefficient (k), were evaluated by spectroscopic ellipsometry using a phase-modulated ellipsometer (UVISEL HR 460, Jobin Yvon-Horiba) in the energy range $0.75-4.50 \mathrm{eV}$ at an incidence angle of $70^{\circ}$. The real and imaginary parts of complex refractive index were calculated from the measured ellipsometric angles Psi $(\Psi)$ and Delta $(\triangle)$, by fitting of the modelled Psi and Delta spectra to the experimental ones and using an appropriate sample structure model. The films were thick enough so that interference effects at the film/substrate interface could be neglected.

\section{Computational details}

The "ab initio" calculations were performed within the DFT framework [6], using the pseudopotential method with the projector augmented wave (PAW) [7] potentials as implemented in the VASP code [8-10]. For the exchange-correlation energy was used the local density approximation (LDA) [11]. A Cut-off energy (Ecut) of $500 \mathrm{eV}$ was used for the plane-wave basis expansion. Brillouin zone (BZ) integrations were carried out using the Monkhorst-Pack scheme [12] with a mesh of 7x7x7 points in the reciprocal unit cell. This number of points is reduced by symmetry to a set of points in the irreducible part of Brillouin zone.

The optical properties of stoichiometric compounds, $\mathrm{TiC}_{\mathrm{x}} \mathrm{O}_{1-\mathrm{x}}$, were calculated using 64 atoms periodic supercells in the rocksalt structure with $\mathrm{x}=0,0.25,0.5$, 
0.75 , 1 . The interband contribution to the dielectric function, $\varepsilon(\omega)=\varepsilon_{1}+i \varepsilon_{2}$, was obtained taking in account all the allowed direct transitions from the occupied to unoccupied states in the calculation of the imaginary part $\left(\varepsilon_{2}\right)$ and then, by using Kramers-Kronig relations [13], the real part is estimated. We also calculated the real part of the optical conductivity

$$
\sigma(\omega)=-i \frac{\omega \varepsilon_{2}}{4 \pi}
$$

which in this case accounts only for optical absorption due to direct interband transitions.

The contribution of intraband transitions to the optical properties can be calculated using the classical Drude model [14]

$$
\varepsilon(\hbar \omega)=\varepsilon_{1}+i \varepsilon_{2}=\varepsilon_{\infty}-\frac{\left(\hbar \omega_{p}\right)^{2}}{\Gamma^{2}+(\hbar \omega)^{2}}+i \frac{\left(\hbar \omega_{p}\right)^{2} \Gamma}{\hbar \omega\left(\Gamma^{2}+(\hbar \omega)^{2}\right)}
$$

where $\varepsilon_{\infty}$ represents the dielectric response at large photon energies, $\Gamma=\hbar / \tau$ is the damping constant related to the charge carrier scattering time $(\tau)$ and $\hbar \omega_{p}$ is the unscreened plasma energy

$$
\hbar \omega_{p}=\sqrt{\frac{e^{2} \hbar^{2} N_{f}}{\varepsilon_{0} m^{*}}}
$$

with $\mathrm{N}_{\mathrm{f}}$ and $\mathrm{m}^{*}$ being the density and effective mass of the nearly free electrons, respectively.

\section{Results and discussion}

\section{I. composition and structural features}

Table I summarizes the chemical composition and the main structural parameters for the films produced at different oxygen flow. X-ray diffraction analysis shows that all the films crystallized in a fcc rock-salt, though defective structure. All the analyzed films have metalliclike appearances, with values of composition ratios, $\mathrm{C}_{\mathrm{O}} / \mathrm{C}_{\mathrm{Ti}}$ between $\sim 0.17$ and 1.44 and $\left(\mathrm{C}_{\mathrm{O}}+\mathrm{C}_{\mathrm{C}}\right) / \mathrm{C}_{\mathrm{Ti}}$ between about 0.5 and 1.6. The diffraction patterns suggest that the films crystallize in a $\mathrm{B} 1-\mathrm{NaCl}$ crystal structure, whose nature is quite difficult to an-ambiguously 
determine due to the very close peak positions of both TiC (ICDD card $n^{\circ} 32-1383$ ) and $\mathrm{TiO}$ (ICDD card n 77-2170). Anyway, the oxygen addition to the growing structures (fcc TiC) is highly probable since the formation of a titanium-oxygen bond is energetically more favorable than that of titanium with carbon. Thus, and though the low oxygen content may not be enough to form $\mathrm{TiO}$, the fcc lattice is most likely to result from a highly sub-stoichiometric TiC-type lattice, where oxygen occupies some of the $C$ vacancies, forming a kind of $\mathrm{Ti}(\mathrm{C}, \mathrm{O})$ solid solution-type structure. The lattice parameters evolution show a first increase from $0.4216 \mathrm{~nm}\left(\mathrm{TiC}_{0.32} \mathrm{O}_{0.17}\right)$ to $0.4228 \mathrm{~nm}\left(\mathrm{TiC}_{0.36} \mathrm{O}_{0.45}\right)$, which is probably due to the oxygen filling of vacant positions in the highly sub-stroichiometric $\mathrm{TiC}_{\mathrm{x}}$ film represented by the first sample $\left.\left(\mathrm{C}_{\mathrm{O}}+\mathrm{C}_{\mathrm{C}}\right) / \mathrm{C}_{\mathrm{Ti}} \approx 0.5\right)$. For these first samples we also observe an increase in the grain size, and thus there is an increase in the number of $\mathrm{Ti}$ atoms belonging to the grain that are placed at the grain surface (corresponding to unsaturated Ti bonds) as previously suggested by P.E. Schmid et al. [15]. Increasing oxygen content, the lattice parameters decrease progressively, from $0.422 \mathrm{~nm}\left(\mathrm{TiC}_{0.24} \mathrm{O}_{0.78}\right)$, to $0.4212 \mathrm{~nm}\left(\mathrm{TiC}_{0.22} \mathrm{O}_{1.13}\right)$ and $0.4179 \mathrm{~nm}\left(\mathrm{TiC}_{0.19} \mathrm{O}_{1.44}\right)$. This decrease is now most likely due to the progressive replacement of carbon by oxygen in non metal sub-lattice. In fact, the ratio $\left(\mathrm{C}_{\mathrm{O}}+\mathrm{C}_{\mathrm{C}}\right) / \mathrm{C}_{\mathrm{Ti}}$ is now above 1 and thus the more reactive element (oxygen) is probably increasing its presence in the fcc lattice. Nevertheless, these results are in general good agreement with the values in the literature $[5,14]$. For oxygen rich films the lattice parameter is higher than the reference bulk value, which can be attributed to the presence of some carbon in the non-metal sublattice. It is also known that in oxygen rich compounds, about $10 \%$ of sites in the metal and non-metal sublattice can be vacant [16].

\section{Optical characteristics}

The real $\left(\varepsilon_{1}\right)$ and imaginary $\left(\varepsilon_{2}\right)$ part of the dielectric function of the different samples, derived directly from the ellipsometric measurements are shown in Figure 1 as a function of the photon energy. There is a clear dependence of dielectric function on the film 
stoichiometry, which is reflected on shift of the screened plasma frequency, $\varepsilon_{1}(\omega)=0$, and in the decrease of $\varepsilon_{2}(\omega)$ as a function of the oxygen content in the film.

The variation of the optical properties of $\mathrm{TiC}_{x} O_{y}$ can be understood by analyzing the electronic partial density of states of $\operatorname{TiC}_{\mathrm{x}} \mathrm{O}_{(1-\mathrm{x})} \mathrm{x}=0 ; 0.5 ; 1$ compounds represented in Figure 2. For TiC, at the Fermi-energy $\left(E_{F}\right)$ (located at the energy value of zero), the contributions from metallic d-orbitals and metalloid p-orbitals are similar and the interband transitions may occur at low energies. This is why the TiC reflectance is almost flat until low energies, not showing the drop in reflectivity characteristic of Drude-like behaviour. In the case of TiO, at $E_{F}$ the metal $d$ partial density of states dominates, with the contribution of the metalloid $p$ states being very small. This means that at low energies, interband transitions must be weak and optical properties are characterized by intraband transitions. This situation is interrupted by an onset of interband transitions at $6 \mathrm{eV}$. They can be identified as transitions between the increasing number of p-states $6 \mathrm{eV}$ below $E_{F}$ and d-states at $E_{F}$. Integrating the DOS between the first minimum below $E_{F}$ and the Fermi level we can estimate the number of electrons/per molecule at $E_{F}$ and the nearly free electron density of the compound. Notice the increase of the number of electrons at $E_{F}$ with the increase of oxygen content, from almost 0.1 electrons/per molecule for TiC to about 2 electrons/per molecule in case of TiO.

Thus, the optical properties of $\mathrm{TiC}_{\mathrm{x}} \mathrm{O}_{\mathrm{y}}$ films at low energies are mainly due to intraband transitions of quasi-free electrons. The optical properties, $\varepsilon_{1}$ and $\varepsilon_{2}$, of the $\mathrm{TiC}_{\mathrm{x}} \mathrm{O}_{\mathrm{y}}$ films were examined using conventional Drude plots, as described in Ref. 17. From these plots the damping constant and the free plasma energy were obtained for all the samples.

Figure 3 shows the free plasma energy $\left(\hbar \omega_{p}\right)$ and the damping constant $(\Gamma)$ as a function of oxygen content. The low value of the plasma energy for carbon rich samples is characteristic of TiC compounds [18], due to low value of density of nearly free electrons (see TiC DOS in fig. 2) in these compounds. It may also be due to the presence of graphitic carbon not bonded to Ti embedded in the film. The initial raise of the free plasma energy with the 
increase of oxygen content can be explained by two competitive effects. On one hand, the filling of empty lattice sites by oxygen leads to a decrease of both the free electrons density and the plasma energy. This process is probably dominated by the effect of the increasing number of Ti atoms localized at the grain surfaces, due to the increase of the grain size, with the consequent raise of the nearly free electron density and plasma energy. This last process is reinforced with carbon substitution by oxygen at the non-metal sublattice for the nearly stoichiometric sample (TiCO), process which as we can see from DOS in fig 2 increases the nearly free electron density. The decrease of free plasma energy with oxygen content for overstoichiometric films, is also correlated with increasing density of defects, either oxygen localized at grain surfaces or Ti vacancies $[15,16]$, leading to a decrease of the free electron density at the Fermi level and as well as plasma energy. This could also be explained by the presence of graphitic carbon not bonded to $\mathrm{Ti}$, which is evidenced by the fact that oxygen is already in excess relative to $\mathrm{Ti}$ and that oxygen bonds preferentially with titanium.

The variation of the damping constant (Figure 3b) with increasing oxygen content, is correlated with density of defects within the film [15]. For substoichiometric films, the increase of oxygen content leads to a decrease of vacancies at non-metal sublattice and a consequent increase in charge carrier scattering time. For overstoichiometric films, there is an increase in film non-stoichiometry and consequently an increase in the density of defects as the oxygen content increases. As a result there is a raise of the damping constant with the increase of oxygen content.

The contribution from intraband transitions to the optical constants of the films was calculated, over the experimental energy range, by using the free plasma energy and damping constant parameters. The total dielectric constant was then separated into contributions from free carriers and bound electrons. The energy-dependent interband optical conductivity, calculated from the imaginary part of experimental dielectric function after subtracting the intraband free carrier contribution, is shown in Figure 4. The experimental optical 
conductivity is in good agreement with our first principles calculations of the optical interband conductivity of stoichiometric $\mathrm{TiC}_{\mathrm{x}} \mathrm{O}_{1-\mathrm{x}}$. Our theoretical results are also in excellent agreement with previous calculations for $\mathrm{TiC}$ and $\mathrm{TiO}$ compounds $[19,20]$. We should notice the overall decrease of the experimental optical absorption with the increase of oxygen content, in agreement with the theoretical results. There is a discrepancy, however, with respect to the onset of strong interband transitions which experimentally is located at $4 \mathrm{eV}$ rather than at $6 \mathrm{eV}$ as predicted by the DFT calculations. These strong interband transitions correspond to transitions between the increasing number of p-states $6 \mathrm{eV}$ below $E_{F}$ and dstates at $E_{F}$ as explained above. Experimentally we do observe strong interband transitions at approximately $1 \mathrm{eV}$, which is also evident in the calculated interband optical conductivity.

\section{Conclusions}

The optical properties of $\mathrm{TiC}_{\mathrm{x}} \mathrm{O}_{\mathrm{y}}$ films have been investigated as a function of the oxygen content in a wide composition range. The dielectric function, directly deduced from spectroscopic ellipsometry measurements, strongly depends on film stoichiometry and chemical composition. In order to separate contributions due to free carriers and to interband transitions, the dielectric function in the low energy region was modeled using the Drude approximation. Our results suggest that for the free plasma energy and the damping constant there are two different regimes associated to the stoichiometry-related defects, one for substoichiometric samples related to Ti localized at grain surfaces, carbon replacement and filling of vacancies by oxygen, and another one for overstoichiometric samples associated with metal vacancies, oxygen localized at grain surfaces and or graphitic carbon at grain boundaries. The experimental interband optical conductivity, obtained from the imaginary part of dielectric function after subtracting the intraband contribution, is in good agreement with the theoretical optical conductivity obtained by first principle calculations. It was demonstrated that the optical properties of $\mathrm{TiC}_{\mathrm{x}} \mathrm{O}_{\mathrm{y}}$ change effectively as a function of the 
oxygen content in the films. Although the produced films may not be stoichiometric, this study provides a novel concept for tuning the optical properties of titanium oxycarbides by adjusting the oxygen flow during the deposition process and consequently the oxygen content within the film.

\section{Acknowledgments}

This work was partially supported by the projects SeARCH (CONC-REEQ/443/EEI/2005) and PoliFilm (PTDC/CTM/69362/2006) funded by the Portuguese Foundation for Science and Technology (FCT) and the European Community Fund FEDER. One of us is also indebt to FCT for the financial support under PhD grant no SFRH / BD / 27569 / 2006. 


\section{References}

[1] J. Yao, J. Shao, H. He, Z. Fan, Vacuum, 81 (2007) 1023.

[2] A.T. Santhenam, Application of transition metal carbides and nitrides in industrial tools, in: S.T. Oyama (Ed.), The Chemistry of Transition Metal Carbides and Nitrides, Blackie Academic and Professional, London, 1996, pp. 28-52.

[3] A. R. Bally, P. Hones, R. Sanjinés, P. E. Schmid, F. Lévy, Surface and Coatings Technology, 108 (1998) 166.

[4] A.C. Fernandes, P. Carvalho, F. Vaz, S. Lanceros-Méndez, A.V. Machado, N.M.G. Parreira, J.F. Pierson, N. Martin, Thin Solid Films, 515 (2006) 866.

[5] L. S. Marques, A. C. Fernandes, F. Vaz, M. M. D. Ramos, Plasma Process. Polym., 4 (2007) S195.

[6] P. Hohenberg, W. Kohn, Phys. Rev., 136 (1964) 864.

[7] P.E. Blochl, Phys. Rev. B, 50 (1994) 953.

[8] G. Kresse and J. Furthmuller, Comput. Math. Sci., 6 (1996) 15.

[9] G. Kresse and J. Furthmuller, Phys. Rev., 54 (1996) 11169.

[10] G. Kresse and J. Hafner, Phys. Rev. B, 47 (1997) 558.

[11] Perdew and Zunger, Phys. Rev. B, 23 (1982) 5048.

[12] J. H. Monkhorst, J. Pack, Phys. Rev.B, 13 (1976) 5188.

[13] C. Kittel, Introduction to Solid State Physics, Wiley, New york 1996, p292.

[14] S. Elliott, The Physics and Chemistry of Solids, Wiley, New york 1998, p379.

[15] P. E. Schmid, M. S. Sunaga, F. Lévy, J. Vac. Sci. Technol. A, 16 (1998) 2870.

[16] C. Leung, M. Weinert, P. B. Allen, R. Wentzcovitch, Phys. Rev. B, 54 (1996) 7857. 
[17] M. Thèye, Phys. Rev B, 2 (1970) 3060.

[18] J. F. Alward, C. Y. Fong, M. El-Batanouny, F. Wooten, Phys Rev B, 12 (1975) 1105.

[19] J. Izquierdo, A. Vega, S. Bouarab, M. A. Khan, Phys. Rev. B, 58 (1998) 3507.

[20] A. Delin, O. Eriksson, R. Ahuja, et al., Phys. Rev. B, 54 (1996) 1673. 